\title{
A case report on incidence of acute pain on right side of the face
}

\author{
Harshita Gupta $^{1 *}$, Sumit Bhateja ${ }^{2}$, Geetika Arora ${ }^{3}$ \\ ${ }^{1}$ Dental Surgeon, ${ }^{2} \mathrm{HOD},{ }^{3}$ Reader, ${ }^{2}$ Dept. of Oral Medicine \& Radiology, ${ }^{\mathbf{3}}$ Dept. of Public Health Dentistry, ${ }^{\mathbf{1} 2}$ Manav Rachna \\ Dental College, Faridabad, Haryana, ${ }^{3}$ Inderprastha Dental College \& Hospital, Ghaziabad, Uttar Pradesh, India
}

\section{*Corresponding Author: Harshita Gupta}

Email: harshitag149@gmail.com

\begin{abstract}
Trigeminal Neuralgia is a nerve disorder of the 5th cranial nerve, i.e. Trigeminal nerve. It is one of the most painful disorders to plague humans since at least as far back as 1st century A. D. Even more than that trigeminal neuralgia does not have any visible signs or causes, so for a long time they went untreated. Now, with advanced imaging procedures such as MRI, it can be diagnosed and managed. The various diagnostic and management methods of Trigeminal Neuralgia are mentioned below.
\end{abstract}

Keywords: Trigeminal Neuralgia, Case Report, History, Classification, Diagnostic Grading, White and Sweet Criterion, Differential Diagnosis, Management.

\section{Introduction}

Trigeminal Neuralgia is a peripheral painful neuropathy. Neuropathic pain is any pain that is caused due to a lesion or disease of the somatosensory nervous system. There are over 100 types of neuropathies recorded, of which trigeminal neuralgia is one. They maybe mononeuropathies, i.e. damage to a single nerve or polyneuropathies, i.e. symmetrical and bilateral injury with sensory loss, weakness and burning; due to axonal or myelin injury. They can be spontaneous with episodic or continuous temporal natures. Trigeminal neuralgia has characteristic episodic paroxysms with uncommon tactile and thermal sensations such as numbness, tingling, pins and needle sensations, burning, shooting or electric shock-like pains. It can also be evoked by stimulating an abnormally sensitive area, resulting in a sequence of spontaneous attacks via ectopic sensory discharges on mechanosensitive neural pathways, like in Tinel Sign. ${ }^{1,2}$

\section{Case Report}

A male 50- year old patient walked into the OPD with chief complaint of severe pain in right maxillary posterior region since past 5 days. He revealed that he has been experiencing unbearable, severe, pricking, electric, unilateral pain that exacerbates while chewing, swallowing, speaking, washing his face, clenching his teeth and even touching. He described the pain to be occurring in episodes, each of which lasted 5-7 minutes. Patient tried to self-medicate using over the counter pain medication which brought mild relief to him. The patient's initial VAS before treatment was 8 .

Patient revealed no significant medical history, and his blood pressure was found normal. Patient was otherwise healthy, moderately nourished, and married with kids. He had ideal erect static and dynamic posture, along with a normal gait with aesthetic build and mesomorphic physique. The patient had a mesocephalic mesoprosopic grossly symmetrical face. He had competent lips, with a prominent chin and normal nasal shape, size and contour. The patient had normal non-palpable cervical lymph nodes and normal TMJ relation.

On extraoral palpation, patient expressed pain on touching supraorbital, infraorbital, zygomatic upper lip and pre-auricular region on right side of face. There was no facial swelling, redness or discoloration.

On intraoral examination patient was found to have 29 teeth with normal generalized wear of tooth crown structure, which was attributed to generalized attrition. The patient was found to have caries in 38 and 16, along with a missing 37, 47 and 17. To exclude a radicular pathology as the cause of pain, TOP (Tenderness on Percussion) of the tooth was checked, which was absent. For further confirmation, 
an OPG was taken. The patient also had Class II deposits of stains and calculus.

Radiographic examination via OPG revealed no radicular pathology and the length of styloid process was found to be normal which ruled out Eagle's syndrome. A tentative provisional diagnosis of trigeminal neuralgia was assigned to the patient.

To confirm this diagnosis, a diagnostic nerve block of first, the mandibular division of the trigeminal nerve, followed by maxillary division of the trigeminal nerve was given to the patient. The patient became immediately free of pain upon receiving the block of maxillary division, thus confirming a diagnosis of trigeminal neuralgia of the maxillary division of trigeminal nerve on the right side.

The patient was prescribed carbamazepine $200 \mathrm{mg}$ t.i.d. for 15 days. After this treatment, patient's VAS was reduced to a 3, which proved the effectiveness of the treatment, so he was asked to continue this treatment thereafter. He was asked for followup every month for regular checkup and for blood investigations to detect long-term side-effects of the drugs.

\section{Case Discussion}

Trigeminal neuralgia has been a common malady since centuries, with the first recorded cases seen in writings of Aretaeus of Cappodocia in the 2nd century A.D. The first detailed documentation of this was done by Nicholas André in 1756. He also coined the term 'tic douloureux' as he made an association between facial tics and pain. ${ }^{3}$ The diagnosis of $\mathrm{TN}$ is made on the basis of a detailed patient history with a large emphasis on pain characteristics. The hallmark pain should be agonizing, paroxysmal and lancinating. International association for study of pain (IASP) has defined trigeminal neuralgia as "sudden usually unilateral severe brief stabbing recurrent pain in the distribution of one or more branches of $5^{\text {th }}$ cranial nerve". International Headache Society (IHS) has defined it as "pain unilateral afflicton of face characterized by brief electric shock like pain limited to the distribution of one or more divisions of trigeminal nerve. ${ }^{4}$

According to the international headache society (IHS), trigeminal neuralgia can be classified as: $:^{5}$

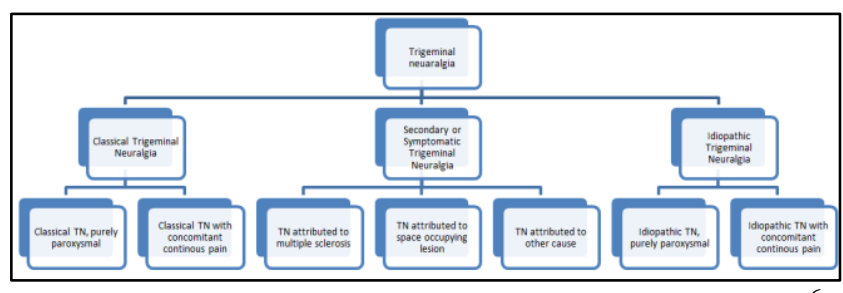

The diagnostic grading for TN can be established as: ${ }^{6}$

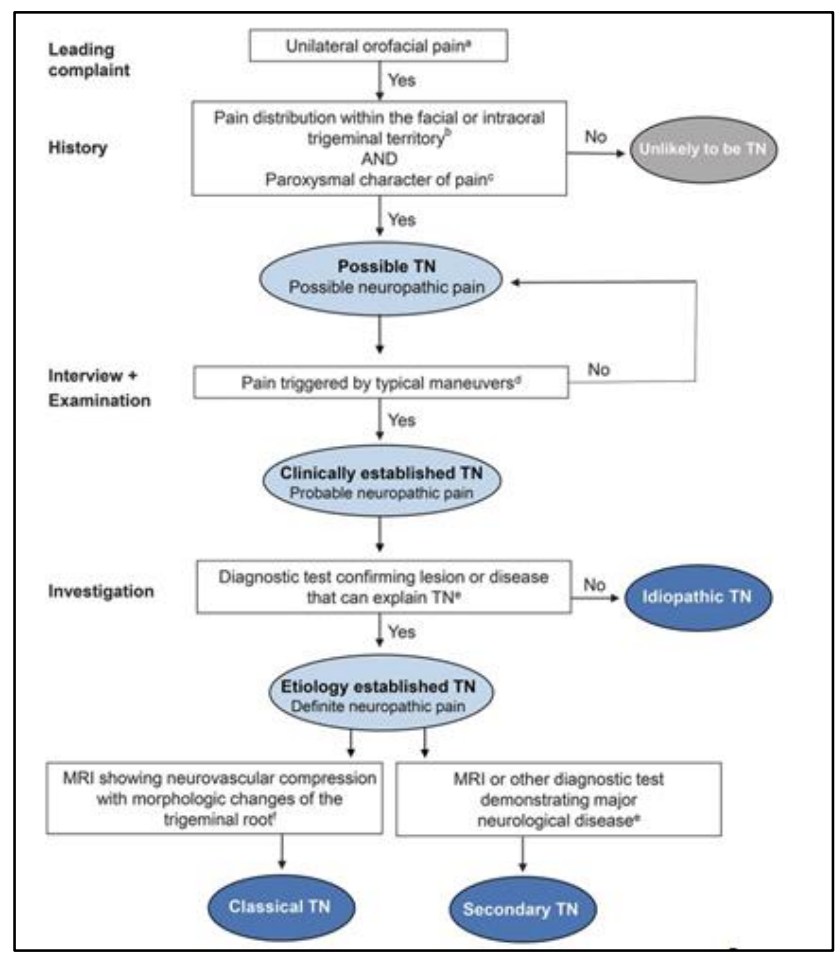

A main proponent for diagnosis of trigeminal neuralgia is the 'White and Sweet criteria' ${ }^{7}$

1. Pain should be paroxysmal.

2. Pain maybe provoked by light touch to the face (trigger zones).

3. Pain is confined to trigeminal distribution.

4. Pain is unilateral.

5. The clinical sensory examination is normal.

The differential diagnoses associated with trigeminal neuralgia are: ${ }^{8}$

Migraine, cluster headaches and atypical facial pain Migraine and cluster headches are not triggered by movement, nor do they respond to carbamazepine. Atypical facial pain is not episodic and can last from hours to days.

Glossopharyngeal neuralgia and occipital neuralgia syndromes - Glossopharyngeal neuralgia 
causes pain in the tonsillar fossa, posterior pharynx, and ear and may be initiated by coughing, yawning, or swallowing cold liquids. Occipital neuralgia causes pain in the posterior head region.

Paroxysmal hemicrania syndrome - Paroxysmal hemicrania syndromes typically last only seconds and occurs in and around either eye.

The first line of treatment for $\mathrm{TN}$ in medical intervention is formed by oral medication, such as carbamazepine and oxycarbamazepine, due its noninvasiveness, low cost and high efficiency. Most commonly used anticonvulsants include carbamazepine, oxycarbamazepine, baclofen, gabapentin, valproic acid, phenytoin, topiramate and lamotrigine, either standalone or in some combnination. Some of the side-effects associated with them are nausea, dizziness, confusion, drowsiness, vision problems, suicidal thoughts and allergies. Carbamazepine can cause liver dysfunction while the most common side-effect of oxycarbamazepine is hyponatremia.

Surgical intervention can be done to relieve the pressure from offending vessel compressing the trigeminal nerve or in cases where surgery can stop the uncontrollable pain by damaging the trigeminal nerve, which might result in temporary or permanent facial numbness.

There are a number of surgical options available to effectively treat $\mathrm{TN}$ which include percutaneous glycerol rhizotomy (PGR), percutaneous balloon compression (PBC), percutaneous stereotactic radiofrequency thermal rhizotomy (PSRTR), gammaknife radiosurgery (GKR), partial sensory rhizotomy (PSR) and MVD.

PGR, also known as a glycerol injection, is a procedure in which A little quantity of sterile glycerol is injected damaging the trigeminal nerve, and thus blocking the pain signals. The majority of people experience immediate and significant pain relief with PGR, though pain may recur later. A common side effect of PGR is facial tingling or numbness.

$\mathrm{PBC}$ of the trigeminal nerve is a procedure where a hollow needle is used to place a balloon next to the nerve which is then inflated. In almost all the patients there is only a mild sensory loss with immediate pain relief.
PSRTR destroys specifically selected nerve fibres linked to pain by means of electrical currents by means of an electrode which is attached to the nerve root under sedation thereafter the patient awakened from sedation to determine whether they can feel the electrical pulses. The patient is then sedated again and the nerve is destroyed by heating the electrodes. A common side effect of PSRTR will experience some facial numbness afterward.

GKR is a technique that aims a high dose of radiation at the root of the trigeminal nerve, gradually resulting in damage to the nerve. Pain relief will gradually take place over several weeks however the initial pain relief may take a few weeks to effect.

PSR entails making a perforation in the skull and the nerve is severed or at times rubbed, which results in the patient having permanent facial numbness.

MVD is a surgical procedure that entails placing Teflon gauze between the nerve and any offending arteries or veins, effectively separating them away from the nerve. ${ }^{9}$

\section{Source of Funding}

None.

\section{Conflict of Interest}

None.

\section{References}

1. Marchettini P, Lacerenza M, Mauri E, Marangoni C. Painful peripheral neuropathies. Curr Neuropharmacol 2006;4(3):175-81.

2. Barbee Jr. JA. Savoie M, Kew CM, Smith A. Neuropathic pain-A glimpse inside the unabating affliction. Pharmacy Times. 20/3/2018. Available: https://www.pharmacytimes.com/publications/healthsystem-edition/2018/march2018/neuropathic-pain-aglimpse-inside-the-unabating-affliction

3. History of Trigeminal Neuralgia. UT Health San Antonio. Cited: 5/11/2019. Available: https://www.uthscsa.edu/patientcare/physicians/services/trigeminal-neuralgia/historytrigeminal-neuralgia

4. Swathi T. Trigeminal Neuralgia- A Case Report with Review of Literature. SAJ. 2017. Case Report 4:102.

5. IHS classification ICHD-3. Cited: 5/11/2019. Available at: https://ichd-3.org/13-painful-cranial-neuropathies-andother-facial-pains/13-1-trigeminal-neuralgia/13-1-1classical-trigeminal-neuralgia/ 
6. Cruccu G, Finnerup NB, Jensen TS. Trigeminal neuralgia: New classification and diagnostic grading for practice and research. Neurol 2016;87(2):220-228. doi:10.1212/WNL.0000000000002840

7. Trigeminal Neuralgia. Florida Pain and Rehabilitation Center. Cited: 5/11/2019. Available at: https://www.flpnr.com/trigeminal-neuralgia-ocala/

8. Singh MK. Trigeminal Neuralgia Differential Diagnoses. Medscape.com. 2019. Available at: https://emedicine.medscape.com/article/1145144differential

9. Turton M, Malan-Roux P. Trigeminal Neuralgia: case report and literature review. Stromatological Dis Sci 2019;3(7).

How to cite this article: Gupta H, Bhateja S, Arora G. A case report on incidence of acute pain on right side of the face. J PaediatrNurs Sci 2019;2(4):102-5. 